\title{
A Review of Past and Future Trends in Perceptual Anchoring.
}

\author{
Silvia Coradeschi and Amy Loutfi \\ Örebro University \\ Sweden
}

\section{Introduction}

Anchoring is the problem of how to create, and to maintain in time the connection between the symbol- and the signal-level representations of the same physical object. In particular robotic systems with symbolic components need to solve the anchoring problem in order to connect the information present in symbolic form with the sensor data that the robot obtains from the physical world. Previously, solutions to the anchoring problem have been implemented on a system by system basis and could therefore only be applied to restricted domains. However, in recent years, the study of anchoring problem per se has gained an increased interest and an attempt to frame the anchoring problem and provide a theoretical groundwork to dealing with anchoring particularly on artificial systems have been explored. The first definition of anchoring was in (Coradeschi \& Saffiotti, 2000) and a community working on anchoring has been established in a number of workshops and a special journal issue (Coradeschi \& Saffiotti, 2001;2003;2004). In this chapter, we present the latest developments in anchoring and outline the future trends. In addition, a specific framework is outlined and used as an example to illustrate the main challenges to be addressed in perceptual anchoring.

The anchoring problem is concerned with the grounding of symbols that refer to specific object entities such as "a cup" or even more specifically "cup-22". Anchoring is not concerned with the process of grounding general properties such as "blue" or general concepts such as "difficult". This is the symbol grounding problem (Harnad, 1990) and anchoring is rather a subset of symbol grounding that is limited to physical objects. Anchoring must take the flow of continuously changing sensor input into account to allow for object persistence in time and space. Even though some properties of an object may change while others remain static, the symbol-percept correspondence should remain intact and should hold the current and updated information. This dynamic maintenance of information differentiates anchoring from pattern recognition which for the most part does not take into account this dynamic aspect or the presence of symbols. One way to consider persistence is to have an internal structure which reifies the correspondence between symbols and sensor data. Thus, many of the contributions in anchoring focus on this internal representation and its formalism. 


\section{Perceptual anchoring in robotics}
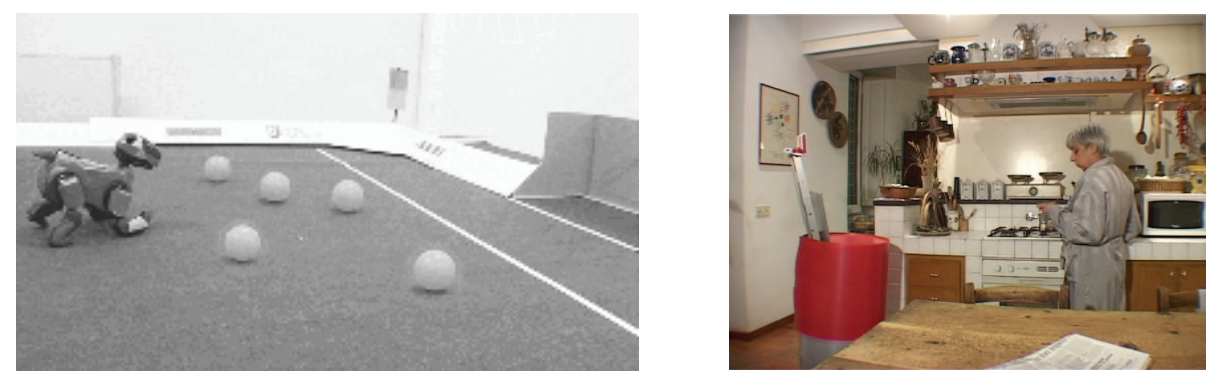

Fig. 1. Examples of anchoring in robotics. (Left) A Robocup domain where similar objects create ambiguities. (Right) Human robot interaction in a home environment where symbolic references to objects are commonly used (photograph by courtesy of Federico Pecora).

Traditionally, anchoring can be seen as a process which creates a shared representation to link several subsystems of an agent, such as the planner to the motion control. In bottom-up approaches, the sensor data determines the initiation of an anchoring process, whereas topdown approaches may initiate an anchoring process upon request. In robotic systems, a number of key challenges are relevant for both bottom-up and top-down anchoring processes. First, uncertainty and ambiguity arise when dealing with real sensors. For these reasons, anchoring may need to consider a number of sub-processes or functionalities which can handle uncertainties and also recover if incorrect decisions are taken. In addition, symbolic descriptions eventually used to link to the perceptual data can be vague and cannot be assessed in terms of a specific quantified sensor value. For instance the concept "a large ball" where the concept large can refer to a range of values whose boundaries are not well defined (Coradeschi et al, 2001). Ambiguous cases can also occur where perceptually similar objects are equally valid candidates in the result of a request. In these cases, further actions may be necessary to resolve the ambiguity (Karlsson et al., 2008). Further, to facilitate human robot interaction, symbols are rarely used in isolation but rather as part of a semantic network where ontological and common sense knowledge plays an important role. As a result, symbolic descriptions may be subject to interpretation and need to handle or cope with variances. For example in Fig. 1, an agent can receive a command to "find a ball", or "find the closest ball" using both definite and indefinite types of references. Further, in scenarios where multiple agents are present, it is important to coordinate and achieve consensus among agents so that a common anchoring is possible.

\section{An example of an anchoring framework}

Here we present an instantiation of an anchoring framework and its core functionalities that illustrate how an anchoring modality works in a real robotic system. Other frameworks have been explored and a discussion of these contributions is given at the end of this section.

The anchoring framework here is based upon (Coradeschi \& Saffiotti, 2000) and contains the following main ingredients:

- A symbol system including: a set $\mathbf{X}=\left\{\mathrm{x}_{1}, \mathrm{x}_{2}, \ldots\right\}$ of individual symbols (variables and constants); a set $\mathbf{P}=\left\{\mathrm{p}_{1}, \mathrm{p}_{2}, \ldots\right\}$ of predicate symbols; and an inference mechanism whose details are not relevant here. 
- A perceptual system including: a set $\Pi=\left\{\Pi_{1}, \Pi_{2}, \ldots\right\}$ of percepts; a set $\Phi=\left\{\varphi_{1}, \varphi_{2}, \ldots\right\}$ of attributes; and perceptual routines. A percept is a structured collection of measurements assumed to originate from the same physical object; an attribute $\varphi_{i}$ is a measurable property of percepts, with values in the domain $\mathbf{D}_{\mathrm{i}}$.

- A predicate grounding relation $\mathrm{g}=\mathbf{P} \times \Phi \times \mathbf{D}$, that embodies the correspondence between unary predicates and values of measurable attributes.

The perceptual system generates percepts and associates each percept with the observed values of a set of measurable attributes and the symbol-percept correspondence is reified in an internal data structure, called an anchor. Since new percepts are generated continuously within the perceptual system, this correspondence is indexed by time.

At every moment $t, \mathrm{a}(\mathrm{t})$ contains: a symbol, meant to denote an object; a percept, generated by observing that object; and a signature, a collection of property values meant to provide the (best) estimate of the values of the observable properties of the object.

To handle anchors, we require functionalities able to create, maintain and remove anchors.

\subsection{Creation of anchors}

The creation of anchors can occur in both a top-down and bottom-up fashion. Bottom-up acquisition is driven by an event originating from a sensing resource (e.g. the recognition of a segmented region in an image) when perceptual information which cannot be associated to any existing anchor is perceived. Top-down acquisition occurs when a symbol needs to be anchored to a percept, such a call may originate from an external user or a top-level module (e.g. planner).

\section{Acquire}

This functionality initiates a new anchor whenever a percept is received which currently does not match any existing anchor. It takes a percept $n$, and return an anchor $\alpha$, defined at $t$ and undefined elsewhere. To make this problem tractable, a priori information is given with regards to which percepts to consider. In bottom-up acquisition, a randomly generated symbol is attributed to the anchor. Furthermore, information about the object and its properties are included into the world model used by the planner, in this way the object can be reasoned about and acted upon.

Find

Takes a symbol $x$ and a symbolic description and returns an anchor a defined at $t$ (and possibly undefined elsewhere). It checks if existing anchors that have already been created by the Acquire satisfy the symbolic description, and in that case, it selects one. Otherwise, it performs a similar check with existing percepts (in case, the description does not satisfy the constraint of percepts considered by the Acquire). If a matching percept is found an anchor is created. Matching of an anchor or percept can be either partial or complete. It is partial if all the observed properties in the percept or anchor match the description, but there are some properties in the description that have not been observed.

\subsection{Maintenance of anchors}

At each perceptual cycle, when new perceptual information is received, it is important to determine if the new perceptual information should be associated to existing anchors. The following functionality addresses the problem of tracking objects over time.

Track

The track functionality takes an anchor $\alpha$, defined for $t-k$ and extends its definition to $t$. The track assures that the percept pointed to by the anchor is the most recent and adequate 
perceptual representation of the object. We consider that the signatures can be updated as well as replaced but by preserving the anchor structure we affirm the persistence of the object so that it can be used even when the object is out of view. This facilitates the maintenance of information while the robot is moving as well as maintaining a longer term and stable representation of the world on a symbolic level without catering to perceptual glitches.

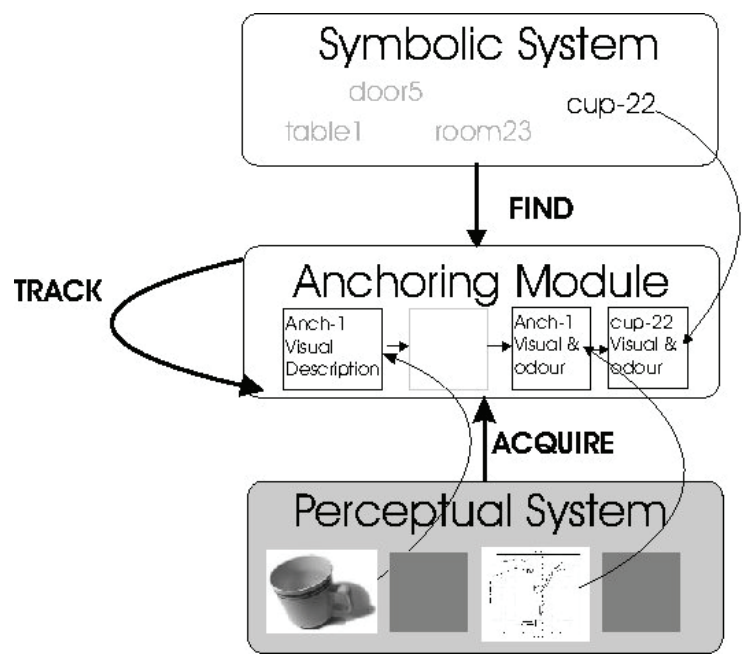

Fig. 2. Graphical illustration of the anchoring functionalities where bottom-up and topdown information is possible and different sensing modalities are used (Louti et. al, 2005).

\subsection{Deletion of anchors}

By having an anchor structure maintained over time, it is possible to preserve the perceptual information even if the object is not currently perceived (caused by the object being out of view and/or by the inaccuracy in the measurement of perceptual data). The challenge is to determine if the association of new percepts is justified or whether certain anchors should be removed. Mechanisms for destroying invalid anchors need to be in place. This is a difficult problem, because conceptually it is not clear when it is appropriate to remove anchors from the system. Anchors could be removed if they are not relevant for the current task, because the object to which it refers has been physically removed from the environment or the reliability of the perceptual information has expired. Anchors may also need to be removed if they have been associated to invalid perceptual data such as sensory glitches. We currently adopt simple solutions in which objects that are not perceived when expected decrease in a "life" value of the respective anchor. When the anchor has no remaining life, the anchor is removed. The decreasing life of anchors is shown in Fig. 4. A more adequate strategy to handle the maintenance of anchors may also be to include a "long term" memory where anchors may be stored for future use.

\subsection{Integration of the functionalities}

The event-based functionalities are restricted to the Find and Acquire while the Track functionality is regularly called. Fig. 4 shows an overview and an example of the framework 
and its functionalities. In the example in the figure, anchors are created bottom-up from the visual percepts. Later, additional features of that object are required, for example, the olfactory property. These features are stored in the anchor. When a top-down request is sent to the anchor module to find a cup with matching properties denoted by the symbol "cup22 ", the Find functionality anchors the symbol to the perceptual data.

As seen in the figure, properties can be collected at different time points using different modalities. Even when certain perceptual properties are updated, such as the smell property, which may change over time, other perceptual properties are maintained. Conversely, if the visual percepts of an anchor are replaced, the smell property previously obtained is not lost. In this way, the anchor is used to compensate for any dynamically changing features of an object. Furthermore, the perceptual description of anchors can be accessed by the planner to reason about perceptual knowledge. In certain cases, this may result in specific calls to perceptual actions in order to disambiguate between similar objects.

\subsection{Case study}

Here we outline a brief example of how the anchoring module operates within a simple corridor monitoring scenario. In each corridor there may be several objects, in this case garbage cans. The robot automatically toggles between the task of patrolling the corridor, inspecting objects and waiting for commands from the user. Patrolling the corridor involves moving from corridor to corridor in a discovery for new objects and recognition of previous objects. When an inspect is invoked, the robot visits each object collecting the odour property. The inspect is usually autonomously invoked when new objects are detected.

The robot is equipped with several heterogeneous sensing modalities such as a camera, sonar, tactile sensors, and an electronic nose. In this example, the modalities of interest are the vision, and e-nose. The vision component is trained to detect any visual signal matching garbage cans. For each found object we extract a number of properties such as color, size and relative position using different heuristics. The collection of the properties belonging to an object is called a percept.

The electronic nose component is able to classify odours providing a symbolic categorical description (Loutfi et al., 2005). The robot is also able to localize itself within the corridor using odometry and has a number of high level processes such as a planner which reasons about actions and a plan executor which can monitor motion control.

Before we begin to outline the corridor example, let us first examine the structure of an anchor. Fig. 3 shows two anchor structures that have been created bottom-up from a segmented image. The anchoring module updates anchors such that at every moment $t, \mathrm{a}(t)$, contains:

Name - For top-down anchors a name is a symbol denoting the object in the planner (e.g., Silvia's Cup). For anchors that have been created bottom-up the name is initially arbitrary.

Symbolic description In general a symbolic description is given in a top-down fashion, however for bottom-up anchors the symbolic description may also be derived directly from the perceptual information of the object. For example, the odour classification module may populate the symbolic description with the linguistic odour name when classifying an odour associated to a particular object.

Perceptual description The perceptual description is a vector which consists of the important properties of the object such as position (relative and global), colour, shape, and when available the odour signature.

In the figure the two anchors, Gar-36 and Gar-34, are visually similar however Gar-36 currently has an olfactory property. In the current implementation an anchor is "baptised" 
with the name of the percept which initially invoked the bottom-up process. As will be shown in the next example, the percept may be updated but the anchor persists using the tracking functionality.

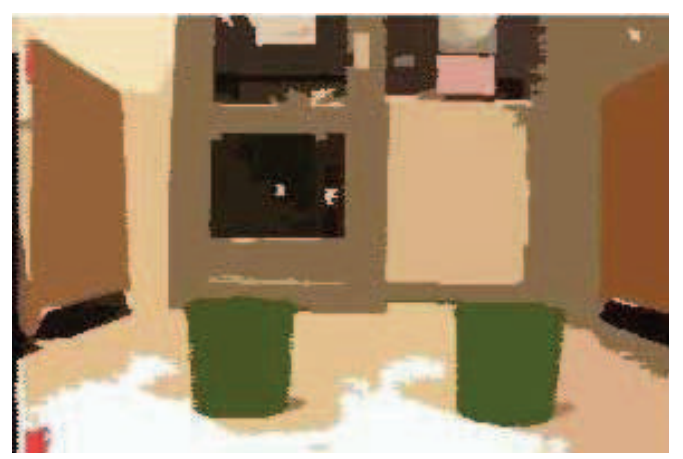

\begin{tabular}{|c|}
\hline $\begin{array}{l}\text { (ANCHOR } \\
\text { :NAME GAR-34 } \\
\text { :ID ANCHOR-6 } \\
\text { :SYMBOLIC-DESCRIPTION }((\text { SHAPE = GARBAGE } \\
\text { CAN) (COLOUR=GREEN) }) \\
\text { :PERCEPTUAL-DESCRIPTION }(<\text { TR GAR- } 3530 \text { [KN } \\
29 \text { GREEN \#1\#GARBAGE L }<1958,347>0 \\
\text { BL }<1958,347>\sim 0: \text { IN-ROOM CORR- } 2: \text { NEC } 0.21000004 \\
: \text { LIFE } 0.8]>\end{array}$ \\
\hline $\begin{array}{l}\text { (ANCHOR } \\
\text { :NAME GAR-36 } \\
\text { :ID ANCHOR-8 } \\
\text { :SYMBOLIC-DESCRIPTION }((\text { SHAPE }=\text { GARBAGE }) \\
\text { (COLOUR=GREEN)(SMELL=ETHANOL)) } \\
\text { :PERCEPTUAL-DESCRIPTION }(<\text { TR GAR-36 } 30 \text { [KN } \\
29 \text { GREEN \#1=GARBAGE L }<1979,-173>\sim 0 \text { BL }<1979,- \\
173>\sim 0: \text { IN-ROOM CORR- } 2: \text { NEC } 0.21000004: \text { LIFE } \\
0.8]>)\end{array}$ \\
\hline
\end{tabular}

Fig. 3. (Left) Segmented image from the vision module observing two green garbage cans at the center of the screen. (Right) The anchors created in a bottom-up manner for each object. Gar-34 refers to the garbage can on the left of the image and Gar-36 refers to the garbage can on the right.

In Fig. 4 The local space of the robot together with the visual image from the camera as well as the creation, deletion and updating of anchors is depicted during a run through the corridor. The figure contains four snapshots described as follows:
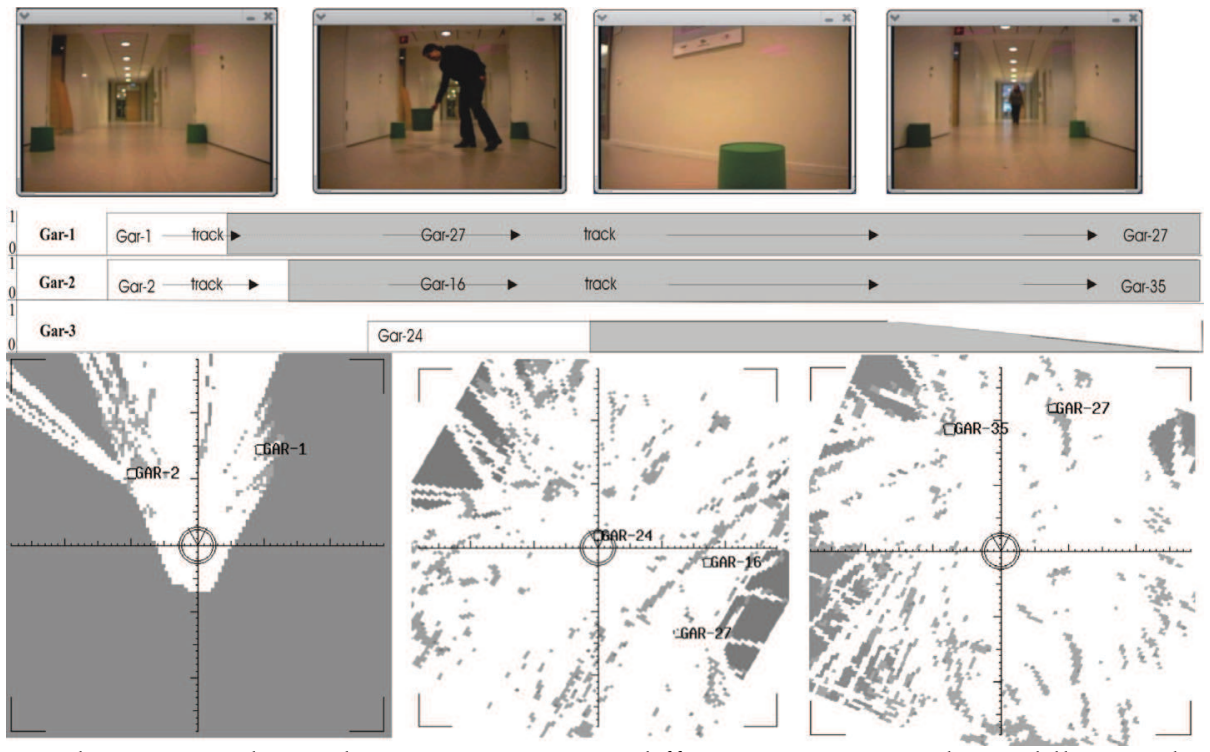

Fig. 4. The top row shows the camera images at different time points, the middle row shows the activity at the anchoring level. Grey bars indicate anchors with olfactory properties. The bottom row shows the corresponding local perceptual space given the changing representation of visual percepts (Loutfi et. al, 2005). 
Scene 1 - The robot begins patrolling the corridor, two visual percepts are detected and two anchors denoted by Gar-1 and Gar-2, are created. An inspect is performed and both anchors obtain olfactory properties, shown in the Figure by the grey colouring. Since the anchors are created in a bottom-up fashion their labels are arbitrary.

Scene 2 - As the robot continues its patrol, another object is inserted into the environment at a later time. Note however, that the previous two anchors are still maintained by the track functionality. Although the local space shows only the current percepts, the anchoring module updates the link between the anchor Gar-1 and the percept Gar-27. A new anchor is also created for the third object denoted by Gar-3 with visual percept Gar-24.

Scene 3 - The robot approaches the object in order to acquire its odour property and the result is stored in the corresponding anchor. Some time later, the object is removed from the environment. The life of the anchor slowly decreases when an expected percept is no longer detected.

Scene 4 - The anchor is removed from the system and unless it is perceived again, its properties cannot be accessed by the find functionalities described above.

This simple scenario shows how the anchoring module is used to create an internal structure which can then maintain the perceptual coherence of objects, considering each object has both spatial and olfactory properties. Even when visual properties of anchors are being updated, the stored smell property remains until a new odour character is acquired by the next inspect action. The previous odour character is then stored in the odour repository.

\subsection{Other approaches to anchoring}

The example above illustrates the main theoretical ingredients necessary for an anchoring module. In the literature, the study of anchoring per se has led to different approaches to address the problem of maintenance and creation of the symbol-percept correspondence referring to objects. Chella et. al, (2003) present a framework where conceptual spaces (Gärdenfors, 2000) are used to combine in a unary formalism all features referring to a specific object, and consequently the combination of the features referring to the object is a single point inside the conceptual space. Similarly, Bonarini et al (2001) have also presented an anchoring framework where concept layer is used to combine features while also using previously established domain knowledge, from a "world modeller". Modayil \& Kuipers (2007) examines unsupervised learning approaches to bootstrap an ontology of objects to sensor input from a robot. Four multiple learning stages are combined in which an object is first individualized, then tracked and described (using shape models) and finally categorized. A collection of works have also extended the anchoring framework beyond the traditional notion of physical objects and contends with: embodied interactions between the robot and objects in the its environment (Chinellato et al, 2007), human movement (Fritsch et al, 2003), actions sequences represented in situation calculus to dynamic properties of objects using conceptual spaces (Chella et al. 2007), perceptually indistinguishable objects (Santore \& Shapiro, 2004).

\section{Cooperative anchoring}

In the previous sections, anchoring has only been considered in the context of single robotic systems. In the case of multiple robotic systems with different and heterogeneous devices cooperating, the anchoring problem undertakes a new complexity. In a distributed system, individual agents may need to anchor objects from perceptual data coming either from sensors embedded directly on the robot or information coming from other devices. Further, agents each with its own anchoring module may need to reach a consensus in order to successfully perform a task. 
Sensor fusion plays an important role for multi-agent or "cooperative" anchoring. A cooperative anchoring approach based on the presented framework has been explored in (LeBlanc \& Saffiotti, 2008) which considers primarily the problem of fusing pieces of information coming from a distributed system. In this work, both complex devices such as mobile robots and simple devices contain pieces of information which may need to be fused together in order to create a global notion of an anchor. Each agent maps items of information into its own anchor space (inspired by Gardenfors' conceptual spaces) where an anchor space is a multi-dimensional domain such as colour, position, weight etc. The individual anchor spaces are mapped into a shared anchor space and from within the shared space information is compared and combined as needed. This is done using the fuzzy intersection of n-dimensional fuzzy sets of the individual anchor spaces. Fig. 5 shows a concrete example where a block is seen from two cameras and an RFID is acquired by an RFID reader. The information is fused in a shared anchoring space using fuzzy sets.

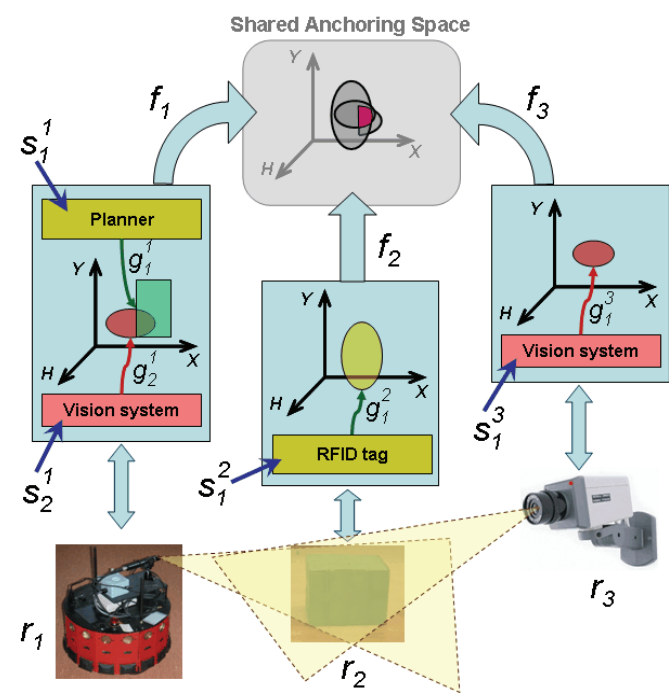

Fig 5. Different elements in a scenario where a mobile robot and a camera mounted in a ceiling detect a parcel and perform cooperative anchoring (courtesy of K. Leblanc, Leblanc \& Saffiotti, 2008).

Another proposed solution for dealing with multi-robot anchoring also extends single-robot systems presented in the previous section. Bonarini et al, (2007) extend their framework to a multi-agent case by combining the information from different agents in a global representation at the conceptual level using a fusion model based on clustering techniques.

Decentralized approaches have also been considered in (Guirnaldo et al, 2004) where each agent has its own anchoring module and broadcasts its anchors to other agents. In this approach, agents have defined roles of leaders and followers and in case of conflict the leader's anchor is accepted, thus it is not clear how fusion would occur if two equally ranked agents conflict. The challenge of achieving an agreement among agents about the objects that are perceived is an open problem. The challenge of reaching an agreement has been studied in the multi-agent community in (Goldman et al, 2007; Kararzyniak \& Pieczynska, 2006). Such work can form the basis for a system where agreement or consensus can be achieved between multiple agents using decentralized anchoring. 


\section{Anchoring for human robot interaction}

Another emerging trend is to study the anchoring process that occurs together with human operators and users. Anchoring is specially suited to HRI application since the symbolic level has clear benefit while communicating with non-experts. Communicate about objects is often central in HRI and such communication requires a coordinated symbol-percept link between human and robot.

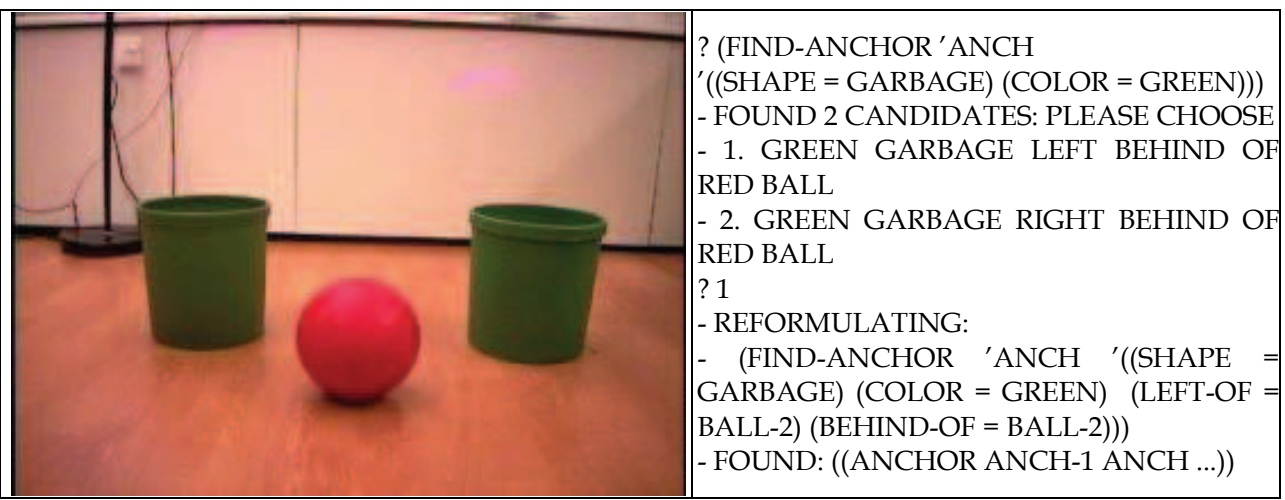

Fig 6. Spatial Relations used to resolve ambiguous situations.

A dialogue system for human-robot collaborations is a particular instance of the anchoring problem, when dialogue about physical objects is concerned. An example of such a dialogue system is explored in (Kruijff \& Brenner, 2007), there information about the object state as well as a history of the object state is used to describe changes in a scene. An important feature of this approach is that it considers descriptions that contain spatial relations among the objects. Spatial relations are crucial when human describe and recognize objects. While communication among devices can be based on coordinates this is not meaningful when the communication is with humans. Further works on using spatial relations and computation of spatial relations between anchors for human robot interaction was explored in (Melchert et. al, 2007). In this work, the spatial relations were used to provide meaningful object descriptions but also could facilitate human participation in the anchoring by using human interaction in the disambiguation process between visually similar anchors. In Fig. 6, an example is shown where a request to find a green garbage can is sent to the anchoring system. The anchoring system cannot disambiguate between the two identical garbage cans and ask the user if he means garbage can on the left and behind the red ball or the one on the right. The user selects the first option and a new request containing the additional information is sent to the anchoring module that succeeds in finding the object. The returned descriptions for the spatial relations of objects present all possible relations of objects. For cases of HRI it would be more beneficial to generate object descriptions with salient and relevant information for the human users (Jordan \& Walker, 2005) .

Other works which examine human participation in the anchoring process include $(\mathrm{Yu} \&$ Ballard, 2004). Here a learning approach is used where spoken names of objects are grounded to image data representing the object. Similarly (Roy, 2005) explores a theoretical framework for involving human participation in the grounding of language to both perception and action using a manipulator robot. 


\section{Anchoring in symbiotic robotic system}

Symbiotic robotic systems are an emerging trend in robotics that combine many of the ingredients in the previous two sections, namely many devices operating in parallel and human users interacting with the system (Coradeschi \& Saffiotti, 2006). The advantage of the symbiotic robotic systems is that many of the current challenges in robots can be circumvented, for instance localization can be helped by cameras on the ceiling and an id of an object can be provided by en RFID tag. However, a symbiotic system require a solution of the two anchoring problems just mentioned, that is, cooperative anchoring and anchoring in cooperation with humans with the additional difficulty that the solutions should be compatible and guarantee a coherent anchoring process. Consider as an example the following scenario:

"Johanna, an elderly woman living alone in her apartment, has a medical condition which affects her blood pressure. Suddenly, while cooking, Johanna feels faint and must sit down. She signals to Emil, her domestic robot, and asks where she last left her blood pressure medicine. Emil communicates with other devices in the home and a camera in the bedroom detects a small bottle on the bedside table. To recognize whether this is the correct medicine, the vacuum cleaner robot already present in the bedroom, is sent to the bedside table. The bottle is successfully recognized as Orvaten (used to treat Johanna's hypotension) using the RFID reader on the vacuum cleaner robot. Emil tells Johanna that the medicine is on the bedside table and Johanna then asks Emil to fetch the medicine for her."

In this scenario, the information from the camera and the RFID reader needs to be combined to recognize the correct medicine and an anchor needs to be established that connects "the blood pressure medicine of Johanna" with the sensor data corresponding to the object and coming from the different devices. The position of the object is also stored and can be then used by Emil to get the medicine. An important challenge in symbiotic systems is the establishment of a shared ontology where concepts referring to objects are coherent between agents, robots, pervasive devices and most importantly the human users. Such ontology forms the basis of the communication among the participant in the anchoring process and provides additional information that can be used in the anchoring process such as function of objects, how different part of objects are related and classes and subclasses of objects. For example, Orvaten is used to treat hypotension, is inside a bottle with an etiquette, and is a subclass of medication. Generate object descriptions that are both meaningful to a specific agent and salient to a task is also essential in systems where different actors are present. In the scenario, the most useful description to Johanna is that the medicine is on the bedside table; while Emil who fetches the medicine may need the actual color and shape of the bottle.

The study of anchoring within the symbiotic system has been examined in a few cases. In Mastrogiovanni et al. (2007) a symbolic data fusion system for an ambient intelligent environment is presented consisting of several cognitive agents with different capabilities. Lopes et al. (2002) describe a way to utilize the KRR component for knowledge acquisition and information disambiguation. Similarly, in (Melchert et al. 2007) we have also examined how KRR system such as LOOM can be integrated into an anchoring framework in the context of the symbiotic system for improved cooperation between devices and human users.

\section{Conclusions}

For artificial intelligence to be used as tools for robotic systems, it is important to be able to capitalize on the work in symbolic AI systems. To accomplish this goal, it is necessary to 
connect the symbolic information to the sensory percepts from the robotic system. This chapter has discussed this important aspect especially concerning the symbol-percept correspondence referring to physical objects. This problem has been defined as the anchoring problem and a number of examples of anchoring in practice have been given. Furthermore, three emerging trends for anchoring has been highlighted: cooperative anchoring, anchoring for HRI and anchoring in symbiotic robotic system where greater symbolic processing is used and thus creating additional challenges for anchoring.

\section{References}

A. Bonarini, M. Matteucci, and M. Restelli. Anchoring: do we need new solutions to an old problem or do we have old solutions for a new problem? In Proc. AAAI Fall Symposium on Anchoring Symbols to Sensor Data in Single and Multiple Robot Systems, 2001.

A. Bonarini, M. Matteucci, and M. Restelli. Problems and solutions for anchoring in multirobot applications. Journal of Intelligent and Fuzzy Systems, 18:245-254, 2007.

A. Chella, M. Frixione, and S. Gaglio. Anchoring symbols on conceptual spaces: the case of dynamic scenarios. Robotics and Autonomous Systems, 43(2):175-188(14), 2003.

A. Chella, H. Dindo, and I. Infantino. Imitation learning and anchoring through conceptual spaces. Applied Artificial Intelligence, 21(4\&5):343-359, 2007.

E. Chinellato, A. Morales, E. Cervera, and A. Del Pobil. Symbol grounding through robotic manipulation in cognitive systems. Robotics and Autonomous Systems, 55(12):851859, 2007.

S. Coradeschi, D. Driankov, L. Karlsson, and A. Saffiotti. Fuzzy anchoring. In Proc of the IEEE Intl Conf on Fuzzy Systems, pages 111-114, Melbourne, AU, 2001.

S. Coradeschi and A. Saffiotti. Anchoring symbols to sensor data: preliminary report. In Proc. of the 17th American Association for Artificial Intelligence Conf. (AAAI), pages 129-135, 2000.

S. Coradeschi and A. Saffiotti, editors. Anchoring Symbols to Sensor Data in Single and Multiple Robot Systems: Papers from the AAAI Fall Symposium. AAAI Press, Menlo Park, California, 2001.

S. Coradeschi and A. Saffiotti. An introduction to the anchoring problem. Robotics and Autonomous Systems, 43(2-3):85-96, 2003.

S. Coradeschi and A. Saffiotti, editors. Robotics and Autonomous Systems, special issue on Perceptual Anchoring. Elsevier Science, 2003.

S. Coradeschi and A. Saffiotti, editors. Anchoring symbols to sensor data. Papers from the AAAI Workshop Technical Report WS-04-03. AAAI Press, Menlo Park, California, 2004.

S. Coradeschi and A. Saffiotti. Symbiotic robotic systems: Humans, robots, and smart environments. IEEE Intelligent Systems, 21(3):82-84, 2006.

G. Cortellessa, A. Loutfi, and F. Pecora. An on-going evaluation of domestic robots. In Proc. of the HRI-08 Workshop on Robotic Helpers, pages 87-91, Amsterdam, NL, 2008.

J. Fritsch, M. Kleinehagenbrock, S. Lang, F. Loemker, G. A. Fink, and G. Sagerer. Multimodal anchoring for human-robot-interaction. Robotics and Autonomous Systems, 43(2):133-147(15): 2003.

P. Gärdenfors. Conceptual Spaces: The Geometry of Thought. MIT Press, Cambridge,MA, 2000. 
C. Goldman, M. Allen, and S. Zilberstein. Learning to communicate in a decentralized environment. Autonomous Agents and Multi-Agent Systems, 15(1):47-90, 2007.

S. Guirnaldo, K.Watanabe, and K. Izumi. Enhancing the awareness of decentralized cooperative mobile robots through active perceptual anchoring. International Journal of Control, Automation and Systems, 2:450-462, 2004.

S. Harnad. The symbol grounding problem. Physica D, 42:335-346, 1990.

P. Jordan and M. Walker. Learning content selection rules for generating object descriptions in dialogue. Journal Artif. Intell. Res. (JAIR), 24:157-194, 2005.

L. Karlsson, A. Bouguerra, M. Broxvall, S. Coradeschi, and A. Saffiotti. To secure an anchor a recovery planning approach to ambiguity in perceptual anchoring. AI Communications, 21(1):1-14, 2008.

R. Katarzyniak and A. Pieczynska. The outline of the strategy for solving knowledge inconsistencies in a process of agents' opinions integration. In 6th International Conference Computational Science, Volume 3993 of Lecture Notes in Computer Science, pages 891-894, 2006.

G. Kruijff and M. Brenner. Modelling spatio-temporal comprehension in situated humanrobot dialogue as reasoning about intentions and plans. In Symposium on Intentions in Intelligent Systems, AAAI Spring Symposium Series, 2007.

K. LeBlanc and A. Saffiotti. Cooperative anchoring in heterogeneous multi-robot systems. In Proc. of the IEEE Int. Conf. on Robotics and Automation (ICRA), Pasadena, CA, 2008.

L.S. Lopes. Carl: from situated activity to language level interaction and learning. In Proc. Intl. Conf. on Intelligent Robots and Systems, pages 890-896, Lausanne, 2002.

A. Loutfi, M. Broxvall, S. Coradeschi, and L. Karlsson. Object recognition: A new application for smelling robots. Robotics and Autonomous Systems, 52:272-289, 2005.

A. Loutfi, S. Coradeschi and A. Saffiotti. Maintaining Coherent Perceptual Information Using Anchoring. In Proc. of the Nineteenth International Joint Conference on Artificial Intelligence. 2005.

F. Mastrogiovanni, A. Sgorbissa, and R. Zaccaria. A distributed architecture for symbolic data fusion. In Proceedings of 20th International Joint Conference on Artificial Intelligence, Hyderabad, India, 2007.

J. Melchert, S. Coradeschi, and A. Loutfi. Knowledge representation and reasoning for Perceptual anchoring. In 19th IEEE International Conference on Tools with Artificial Intelligence (ICTAI), Patras, Greece, 2007.

J. Melchert, S. Coradeschi, and A. Loutfi. Spatial relations for perceptual anchoring. In Proceedings of AISB'07, AISB Annual Convention, Newcastle upon Tyne, UK, 2007.

J. Modayil and B. Kuipers. Autonomous development of a grounded object ontology by a learning robot. National Conference on Artificial Intelligence (AAAI-07), 2007.

D. Roy. Semiotic Schemas: A Framework for Grounding Language in the Action and Perception. Artificial Intelligence, 167(1-2): 170-205, 2005.

J. Santore and S. Shapiro. Identifying an object that is perceptually indistinguishable from one previously perceived. In Proceedings of the Nineteenth National Conference on Artificial Intelligence, pages 968-969. 2004.

C. Yu and D. Ballard. On the integration of grounding language and learning objects. In Proceedings of the Nineteenth National Conference on Artificial Intelligence, pages 488494, 2004. 


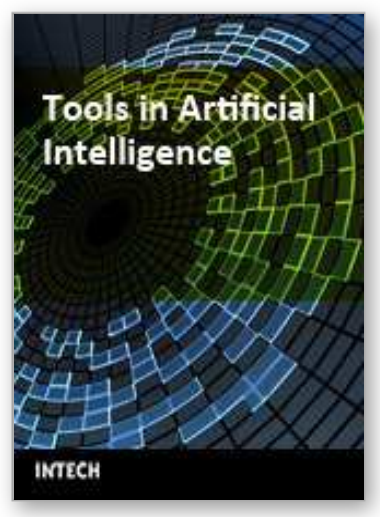

\author{
Tools in Artificial Intelligence \\ Edited by Paula Fritzsche
}

ISBN 978-953-7619-03-9

Hard cover, 488 pages

Publisher InTech

Published online 01, August, 2008

Published in print edition August, 2008

This book offers in 27 chapters a collection of all the technical aspects of specifying, developing, and evaluating the theoretical underpinnings and applied mechanisms of Al tools. Topics covered include neural networks, fuzzy controls, decision trees, rule-based systems, data mining, genetic algorithm and agent systems, among many others. The goal of this book is to show some potential applications and give a partial picture of the current state-of-the-art of Al. Also, it is useful to inspire some future research ideas by identifying potential research directions. It is dedicated to students, researchers and practitioners in this area or in related fields.

\title{
How to reference
}

In order to correctly reference this scholarly work, feel free to copy and paste the following:

Silvia Coradeschi and Amy Loutfi (2008). A Review of Past and Future Trends in Perceptual Anchoring, Tools in Artificial Intelligence, Paula Fritzsche (Ed.), ISBN: 978-953-7619-03-9, InTech, Available from:

http://www.intechopen.com/books/tools_in_artificial_intelligence/a_review_of_past_and_future_trends_in_perc eptual_anchoring

\section{INTECH}

open science | open minds

\section{InTech Europe}

University Campus STeP Ri

Slavka Krautzeka 83/A

51000 Rijeka, Croatia

Phone: +385 (51) 770447

Fax: +385 (51) 686166

www.intechopen.com

\section{InTech China}

Unit 405, Office Block, Hotel Equatorial Shanghai

No.65, Yan An Road (West), Shanghai, 200040, China

中国上海市延安西路65号上海国际贵都大饭店办公楼405单元

Phone: +86-21-62489820

Fax: +86-21-62489821 
(C) 2008 The Author(s). Licensee IntechOpen. This chapter is distributed under the terms of the Creative Commons Attribution-NonCommercialShareAlike-3.0 License, which permits use, distribution and reproduction for non-commercial purposes, provided the original is properly cited and derivative works building on this content are distributed under the same license. 